\title{
Hybrid Electron Holography
}

Cigdem Ozsoy-Keskinbora ${ }^{1}$, Chris B. Boothroyd ${ }^{2}$, Rafal E. Dunin-Borkowski ${ }^{2}$, Peter A. van Aken ${ }^{1}$, Christoph T. Koch ${ }^{3}$

1. Stuttgart Center for Electron Microscopy (StEM), MPI for Intelligent Systems, Stuttgart, Germany.

2. Ernst Ruska-Centre for Microscopy and Spectroscopy with Electrons and Peter Grünberg Institute, Forschungszentrum Jülich, Jülich, Germany.

3. Institute for Experimental Physics, Ulm University, Ulm, Germany.

When a plane wave, whether a photon, electron or neutron, passes through a transparent object, the exit wave provides information about the object's internal structure, refractive index, electric and magnetic fields within and near the specimen plus many other signals, depending on the type of radiation. The ability to detect the full exit wavefunction instead of only the intensity allows for numerical correction of the aberrations of the imaging system. Unfortunately, the phase information of a wave is lost when it hits a detector. Retrieving phase information with high reliability and sensitivity is a key challenge in many fields of optics. Gabor's 1948 holography experiments with electrons showed the potential for reconstructing the complete wavefunction for the first time ${ }^{1}$. Since then, many complementary approaches have been developed in different branches ${ }^{2}$ of optics, each with its own problems ${ }^{3,4}$. In this study, we describe an approach for measuring the full exit wavefunction, which combines two different holography methods to overcome the deficiencies of each technique alone. We combine in-line electron holography (using a setup similar to Gabor's first experiment) to recover high spatial frequency information with an off-axis setup that allows low spatial frequency information to be recovered ${ }^{5}$. The synergistic combination of these two complimentary techniques is achieved by using the off-axis information as an initial guess for the in-line reconstruction algorithm, which then iteratively recovers the phase information. Figure 1 shows an outline of the procedure, which is followed during the reconstruction of the phase by the hybrid method. Initial experiments have been carried out successfully with electrons in a study of iron-carbon core-shell and gold nanoparticles. Figure 2 shows examples of the phase and amplitude that were obtained from several iron-carbon core-shell nanoparticles, even under suboptimal experimental conditions.

\section{References:}

1. Gabor, D., Nature 161 (1948), 777-778.

2. Cowley, J. M., Ultramicroscopy 41 (1992), 335-348.

3. Koch, C. T.; Lubk, A., Ultramicroscopy 110 (2010), 460-471.

4. Latychevskaia, T. et al, Ultramicroscopy 110 (2010), 472-482.

5. Ozsoy-Keskinbora, C. et al, Sci. Rep. 4 (2014), 7020. 


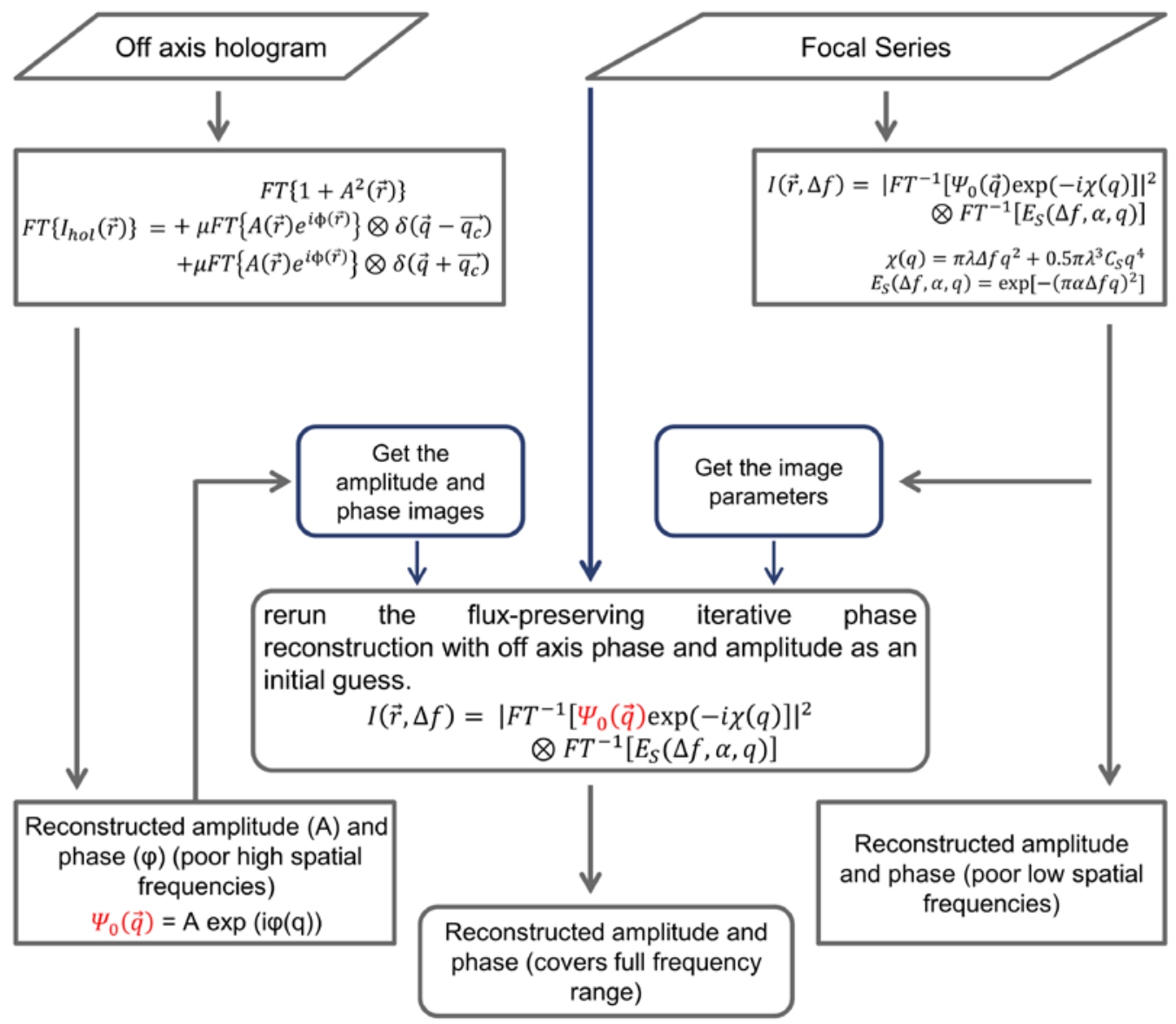

Figure 1. Schematic outline of the wave reconstruction algorithm used in the present work.
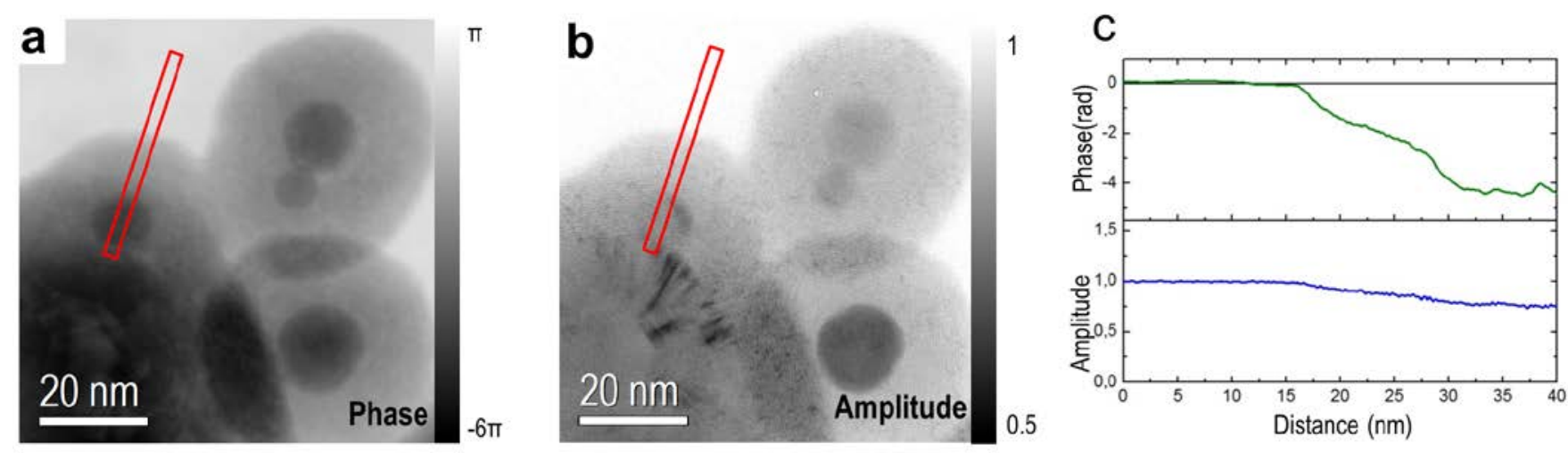

Figure 2. Full exit wavefunction obtained from a sample of iron-filled multi-walled carbon nano-onions using the hybrid method: a) amplitude; b) phase; c) amplitude and background-subtracted phase profiles. 